\title{
ATOMHSTIC STUDIES OF GRAIN BOUNDARIES IN ALLOYS AND COMPOUNDS
}

\author{
Grant No. DE FG02-87ER45295
}
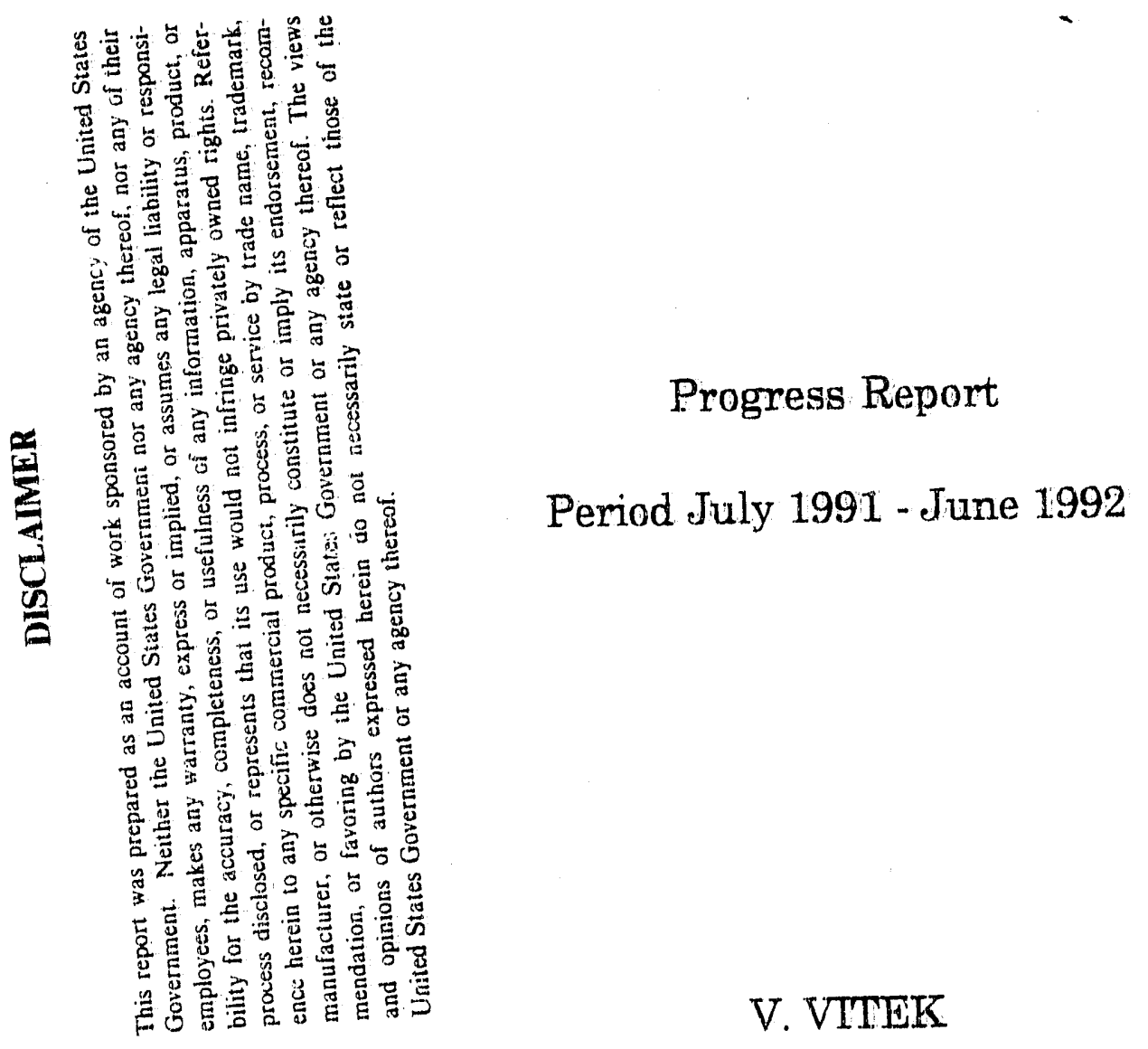

Department of Materials Science and Engineering, University of Pennsylvania, Philadelphia, PA 19104

February 1992 


\section{PERSONNEL}

Professor V. Vitek, Principal Investigator

Ms. Min Yan, Graduate student

Mr. R. Siegl, Graduate student (new)

Dr. M. Sob, Research Associate (until September 1992)

Dr. G. - J. Wang, Post-doctoral Fellow (part-time)

\section{Collaborrators:}

Prof. J. L. Bassani, Department of Mechanical Engineering and Applied mechanics, University of Pennsylrania

Prof. D. Luzzi, Department of Materials Science and Engineering, University of Pennsylvania

Prof. C. J. McMahon, Jr., Department of Materials Science and Engineering, University of Pennsylvania

Dr. S. P. Chen, Los Alamos National Laboratory. 


\section{INTROLUCTION}

In this research project we carry out theoretical, computer modeling, studies of the atomic structure of grain boundaries in binary alloys. Both ordered and disordered alloys are investigated. The goal is to analyze those structural, chemical and electronic features that distinguish alloys from pure metals and are responsible for remarkably different intergranular fracture behavior of alloys when compared with pure metals. The most irnportant phenomenon is, of course, segregation and related structural changes in the boundary region.

When studying segregation phenomena copper-bismuth is a very suitable model: system since bismuth segregation occurs readily, leads to boundary facetting and thus to remarkable changes in the boundary structure, as well as to a very strong embrittlement. Our recent research concentrated on the investigation of the structure of $\Sigma=3(111) /(11 \overline{1})$ facets formed during segregation from boundaries which were originally curved. These Sacets, which in pure copper correspond to coherent twins, contain a high concentration of bismuth in spite of the fact that no segregation takes place to coherent twins if they are present in the sample prior to segregation. This suggests that facetting is associated with formation of a new twodimensional phase and understanding its structure may be the clue to comprehending embrittlement. Fortunately, these facets are ideally suited for high resolution electron microscopy (HREM) and provide an excellent opportunity to carry out a combined experimental and theoretical investigation of their structures. Such an analysis is one of the principal results of our research during this period.

In the case of ordered alloys we explored grain boundaries in $\mathrm{Ni}_{3} \mathrm{Al}$ and $\mathrm{Cu}_{3} \mathrm{~A}$.u with the aim to study the relationship between the ordering tendency, which is quantitatively very different in these two alloys, and grain boundary structure and properties. In both cases we have carried out Nonte-Carlo studies of grain boundaries at finite temperatures for both stoichiometric and non-stoichiometric bulk concentrations. The most interesting finding is a very different behavior of nickel rich and aluminum rich $\mathrm{Ni}_{3} \mathrm{Al}$ which may explain why only nickel rich compounds can be ductilized by boron. These results are described in more detail below.

A large part of our research is expended on the development of descriptions of interatomic forces which are essential for meaningful atomistic studies of interfaces. In fact the construction of the appropriate many-body potentials for the copper-bismuth system was instrumental for the study of boundaries in this system. 
While the methods of construction of many-body potentials were formulated in our previous research, the important new development is that the 'empirical' input are results of ab-initio electronic structure calculations rather than experimental data. In parallel with the development of empirical potentials we have advanced a fully quantum mechanical technique based on the LMTO (linearized muffin-tin orbitals) method. In the framework of this approach electronic and chemical aspects of the interfacial structures and properties can be accounted for. The major problems encountered in this treatment are associated with incomplete self-consistency and deviations trom local spherical symmetry. Approximate but physically justified solution of these problems was the effort during this year.

\section{MANY-BODY POTEINTIALS FOR COPPER-BISIMUTH}

The potentials were constructed within Finnis-Sinclair scheme where the total energy of the system of $\mathrm{N}$ atoms is written as

$$
\boldsymbol{E}=\sum_{i=i}^{N}\left[\sum_{j \neq i} V_{s_{l} s_{j}}\left(\boldsymbol{R}_{y_{j}}\right)-\sqrt{\rho_{s_{i}}}\right]
$$

where

$$
\rho_{s_{1}}=\sum_{j \neq i} \Phi_{s_{i} s_{j}}\left(\boldsymbol{R}_{w_{j}}\right)
$$

Suffices $i$ and $j$ refer to individual atoms and the suffices $\boldsymbol{S}_{i}$ and $\boldsymbol{S}_{j}$ refer to the species of the atoms involved. Both $\boldsymbol{V}$ and $\Phi$ are empirically fitted pair-potentials and summation over $\boldsymbol{j}$ extends over those neighbors of the atom $\boldsymbol{i}$ for which $\boldsymbol{R}_{\boldsymbol{U}}$ is within the cuti-off radii of these potentials. Functions $V_{C u C u}, \mathbf{V}_{B u B v}, \Phi_{C u C u}$, and $\Phi_{B u B t}$ were taken to be the the same as those constructed earlier for pure elements. The function $\Phi_{C u B t}$ was chosen as a geometrical mean of $\Phi_{C u C u}$ and $\Phi_{B t B t}$ which is congruent with its interpretation in terms of hopping integrals. Hence, only the pair potential. $\boldsymbol{V}_{\text {CuBt }}$ needs to be fitted to alloy properties. However, since copper and bismuth do not form any ordered structures and even the solid solution does not exist at higher bismuth concentrations, there are practically no experimental data to be fitted. For this purpose we calculated the total energy of a metastable $L 1_{2}$, f.c.c. based, $\mathrm{Cu}_{3} \mathrm{Bi}$ compound as a function of the volume per atom. This calculation was performed using the all-electron self-consistent full-potential linear-muffin-tinorbital method within the approximation of the local-density. As a result we obtained the equilibrium lattice parameter and the bulk modulus of this compound 
and these were than used as 'empirical' data. Furthermore, the potential was also fitted with the help of Monte Carlo calculations so that it reproduces the enthalpy of mixing for the liquid copper-bismuth solution at $1200 \mathrm{~K}$ which was measured.

\section{STRUCTURE OF THE $\Sigma=3(111) /(1 \overline{1})$ FACETS IN COPPER-BISIMUTH}

As mentioned above, the segregation of bismuth to the curved boundaries corresponding to the $\Sigma=3$ misorientation induces formation of (111)/(11 $\overline{1})$ facets. The HREM analysis of these facets suggests the following model for these interfaces. In the framework of the ' $\mathrm{ABC}$ ' description of the sequence of $\{111\}$ atomic planes in an f.c.c. crystal, the $\Sigma=3(111) /(11 \overline{1})$ twin in pure copper can be represented as

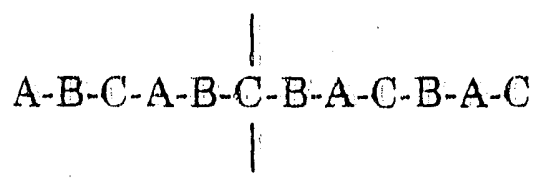

where the vertical lines mark the position of the boundary. The structure containing bismuth can be most appropriately represented as

$$
A-B-C-A-B-C^{\prime}-C(B i)-B-A-C-B-A-C
$$

where $C^{\prime}$ denotes a plane of copper atoms in which one third of the atoms were replaced by hexagonally arranged vacancies, and $\mathrm{C}(\mathrm{Bi})$ the plane of bismuth atoms positioned above these vacancies.

The atomistic modeling of the $\Sigma=3(111) /(11 \overline{1})$ boundary was then carried out using the structure described by (3) as a starting configuration. The relaxation calculation was carried out such that the total energy of the block given by equation (1a) has been minimized with respect to the positions of the individual atoms as well as with respect to the relative rigid body displacement of the two adjoining grains. This displacement has components both parallel and perpendicular to the boundary so that there are neither any tensile nor compressive stresses normal to the boundary nor shear stresses parallel to the boundary present in the final configuration. 
Two distinct structures were found. The first corresponds to the smallest possible boundary period, $\frac{3}{2}[1 \overline{1} 0] \times \frac{1}{2}[11 \overline{2}]$, and the second to $\frac{3}{2}[1 \overline{1} 0] \times[11 \overline{2}]$. The energy of the latter structure is $2: 8 \%$ lower and when the calculations were performed for larger repeat cells this low energy structure has always been obtained. The low energy energy structure is shown in Fig.1 and the high energy structure in Fig. 2. In these figures copper atoms are represented by small circles and the bismuth atoms by large circles and shading distinguishes between atoms belonging to subsequent (1 10$)$ planes separated by the distance $\mid 1 / 4[1 \overline{1} 0] 1$.

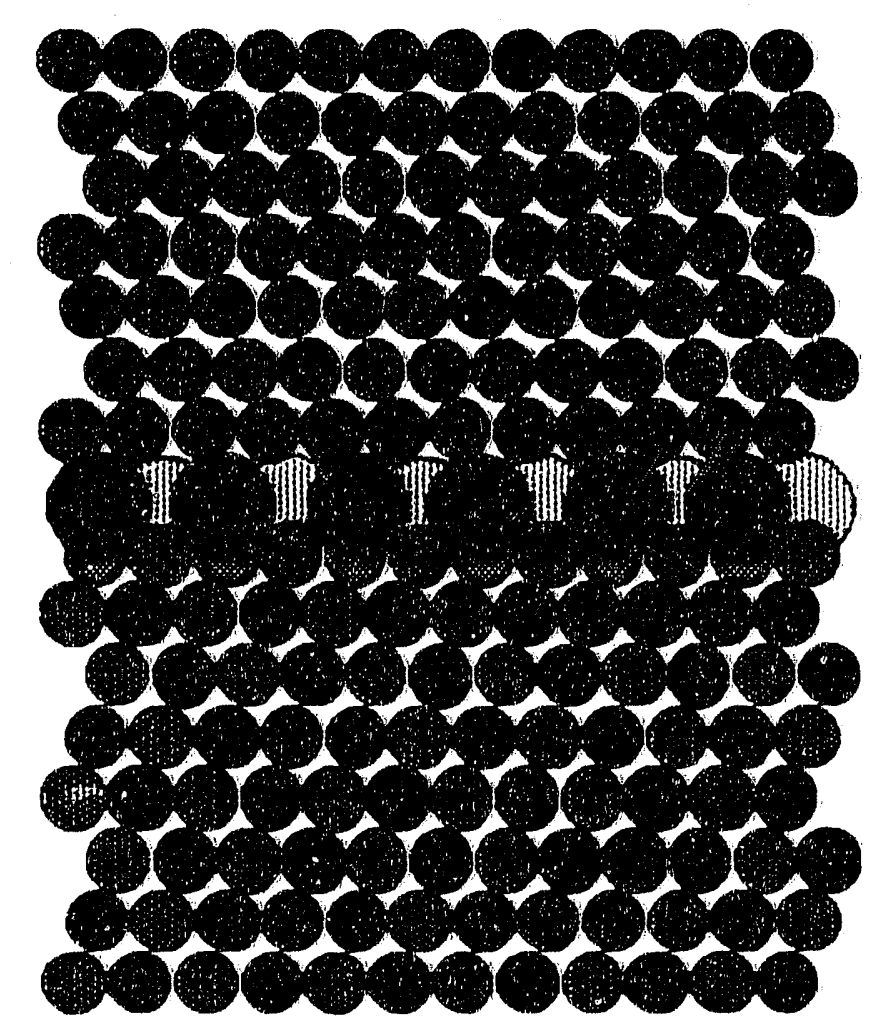

Fig. 1. The low energy structure of the $\Sigma=3(111) /(11 \overline{1})$ boundary.

The overall expansion of the bi-crystal, caused by the rigid body displacement of the two grains across the boundary, is in both structures very close to one interplanar spacing of $\{111\}$ planes in $\mathrm{Cu}$ and the two structures are similar. However, in the low energy structure all the bismuth atoms are contracted towards the plane of copper containing vacancies ( $\left.C^{\prime}\right)$ by $0.5 \AA$ away from the ideal position while in the high energy structure the two bismuth atoms in the repeat cell contract 
towards the plane of copper containing vacancies by $0.93 \mathrm{~A}$ and $0.49 \AA$, respectively. This leads to a significant corrugation of the bismuth layer as seen in Fig.2.

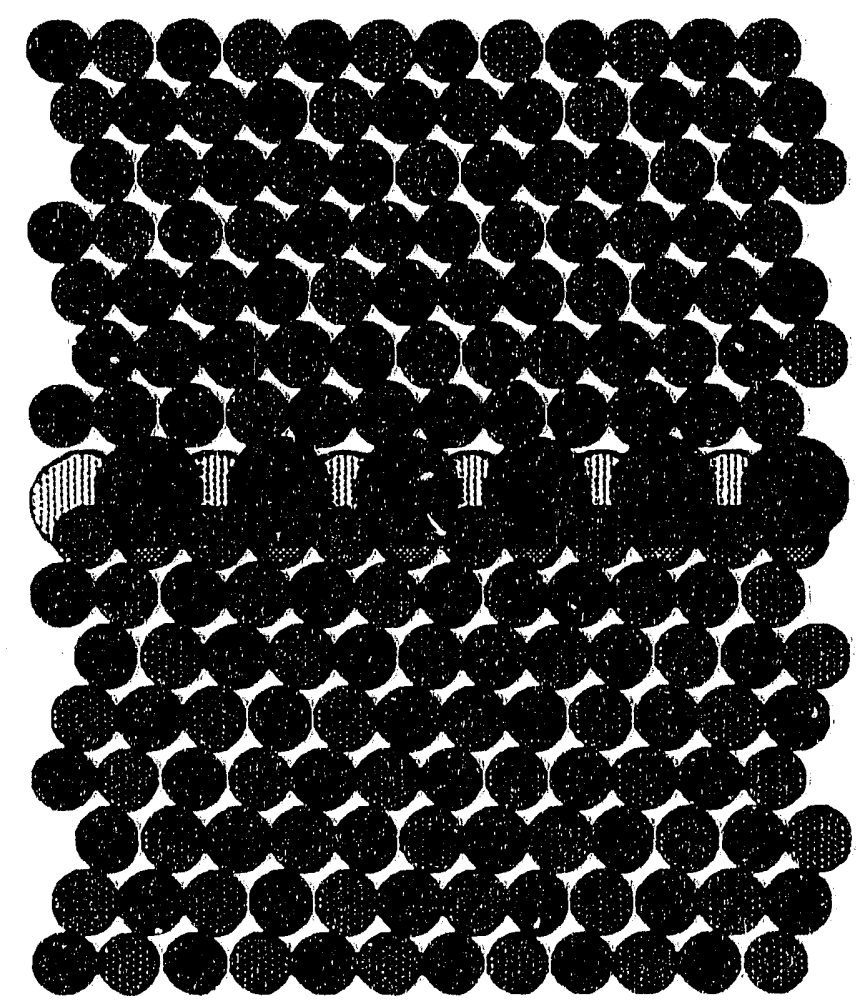

Eig. 2. The high energy structure of the $\Sigma=3(111) /(11 \overline{1})$ boundary.

While the overall featuress of the two structures are similar the differences in the local arrangement of bismuth atoms lead for certain defocus conditions to significantly different HREM images associated with these two structures and it is the low energy structure which is in an excellent agreement with the experimental observation. Details of the comparison between the observed and calculated images can be found in Luzzi et al. (1991) and in the forthcoming paper by Min Yan et al. (1992). These results demonstrate for the first time that HREM studies and theoretical structural analyses can be used synergistically to decipher with a great accuracy the structure of boundaries containing multiple atomic species. At the same time these results provide a firm evidence that the empirical N-body potentials are capable to describe with a sufficient accuracy atomic interactions even in relatively complex systems such as copper-bismuth provided an appropriate and 
sufficient input is used in their construction. For this purpose the self-consistent ab-initio electronic structure calculations are essential since they provide data corresponding to sampling of configurations which are not attainable experimentally:

\section{ORDERED ALLOYS}

The ordered alloys considered are the f.c.c. based $\mathrm{L}_{2}$ alloys $\mathrm{Cu}_{3} \mathrm{Au}$ and $\mathrm{Ni} \mathrm{il}_{3} \mathrm{Al}$. It was found in the earlier studies that in $\mathrm{Cu}_{3} \mathrm{Au}$, which is weakly ordered, the boundary structure is very similar to that in pure f.c.c. materials. On the other hand, in strongly ordered alloys, such as $\mathrm{Ni} 3 \mathrm{Al}$, all the atoms can be regarded as uniquely attached to either the upper or the lower grain so that the ideal $\mathrm{L} \mathrm{l}_{2}$ structure is practically undisturbed on either side of the boundary up to the boundary plane. The reason for these remarkable structural differences can be sought in differences in ordering energies. In the case of a high ordering energy $\left(\mathrm{Ni}_{3} \mathrm{Al}\right)$, the chemical order is the principal controlling factor of the energy of the system. On the other hand, in the case of a low ordering energy ( $\mathrm{Cu}_{3} \mathrm{Au}$ ) more relaxed grain boundary structures are energetically favored.

The preservation of the ideal $\mathrm{Ll}_{2}$ order up to the boundary plane leads to the presence of columns of atomic size cavities in the boundaries in $\mathrm{Ni}_{3} \mathrm{Al}$. These cavities may serve as suitable nuclei for intergranular cracks. Furthermore, when dislocations are being emitted from the crack tip a high energy APB has to be formed at the same time, making the crack blunting more difficult. The atomistic stiudies suggest, therefore, that in strongly ordered $\mathrm{L}_{2}$ alloys the grain boundary structures makes the nucleation and subsequent propagation of intergranular cracks much easier than in weakly ordered $\mathrm{Ll}_{2}$ alloys, such as $\mathrm{Cu}_{3} \mathrm{Au}$.

The present studies focused on effects of temperature and non-stoichiometry upon the boundary structure. For this purpose Monte Carlo calculations have been carried out for various temperatures and stoichiometrieg of the alloys. In the case of $\mathrm{Cu}_{3} \mathrm{Au}$ signifficant compositional disorder has been observed even at room temperature. This suggests that boundaries in this alloy are structurally very similar to those in pure metals or disordered alloys and thus their properties will be similar. In contrast, in the case of stoichiometric $\mathrm{Ni}_{3} \mathrm{Al}$ very little disorder occurs even at temperatures as high as $1200^{\circ} \mathrm{K}$ which close to the meliting temperature. 
Hence, the boundary features described above are preserved even at high temperatures. However, the situation is more complex in mon-stoichiometric $\mathrm{Ni}_{3} \mathrm{Al}$.

As an example we show here the resulits for the $\Sigma 29(520)$ grain boundary in the $\mathrm{Ni}$-rich $\mathrm{Ni}-24 \% \mathrm{Al}$ and $\mathrm{Al}$-rich $\mathrm{Ni}-26 \% \mathrm{Al}$ alloys at $600^{\circ} \mathrm{K}$. Segregation of the excess element towards the grain boundary occurs in both cases but the effect on the boundary structure is very different. The concentration profile of the Ni surpluss in the $\Sigma 29(520)$ grain boundary is shown in Fig.3. As seen from this figure segregation of $\mathrm{Ni}$ occurs and it is accompanied by exchanges between $\mathrm{Ni}$ and $\mathrm{Al}$ atoms in the boundary region. This is also seen in Fig. 4 where shading represents the chemical nature of the atoms. The darkest shade corresponds to $\mathrm{Ni}$ and the lightest to $\mathrm{Al}$. The additional three intermediate levels of shading then represent the probability that an atomic site is occupied by Ni, the darker the shade the higher the probability that $\mathrm{Ni}$ is positioned at the site. It is seen that while the $\mathrm{Ni}$ concentration in the boundary region has increased some sites which were originally occupied by Ni: possess a non-zero probability to be occupied by Al. Hence, the grain boundary becomes chemically disordered as a result of the Ni segregation.

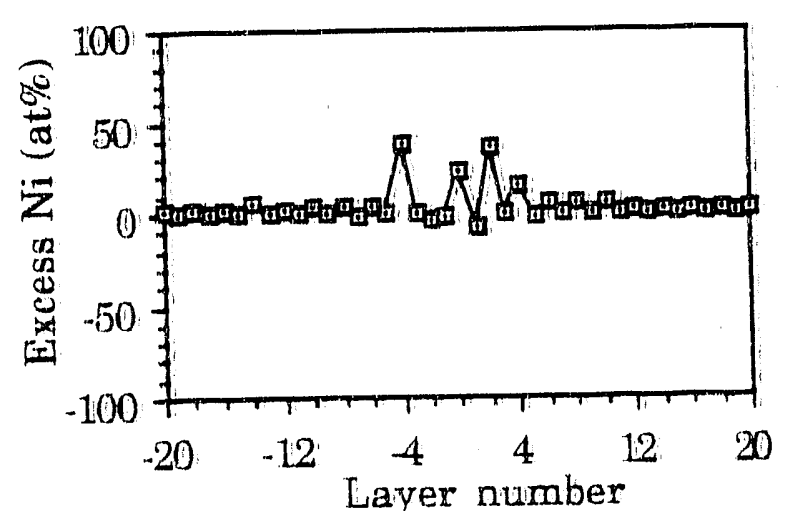

(a)

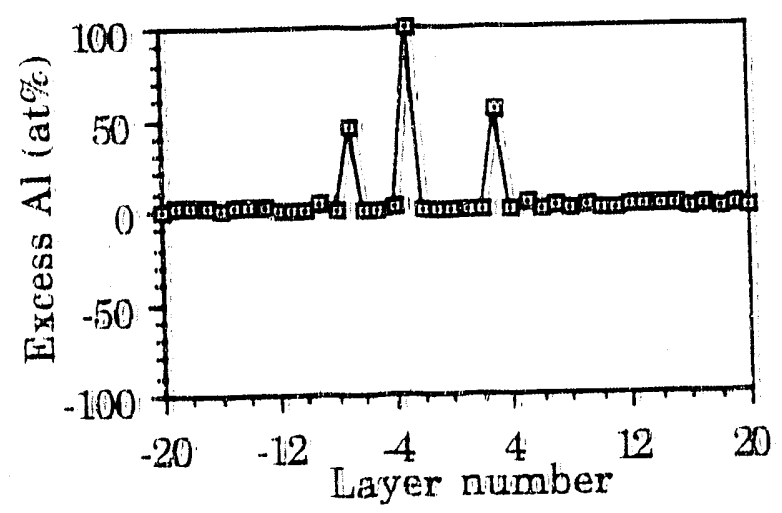

(b)

Fig.3. (a) Excess amounits of $\mathrm{Ni}$ in layers parallel to the boundary plane in the $\Sigma 29(520)$ boundary in $\mathrm{Ni} 3 \mathrm{Al}$ at $600^{\circ} \mathrm{K}$ for bulk composition of $\mathrm{Ni}-24 \% \mathrm{Al}$.

(b) Excess amount of $\mathrm{Al}$ in layers parallel to the boundary plane in $\Sigma 29(520)$ boundary in $\mathrm{Ni} 3 \mathrm{Al}$ at $600^{\circ} \mathrm{K}$ for bulk composition of $\mathrm{Ni}-26 \% \mathrm{Al}$. 


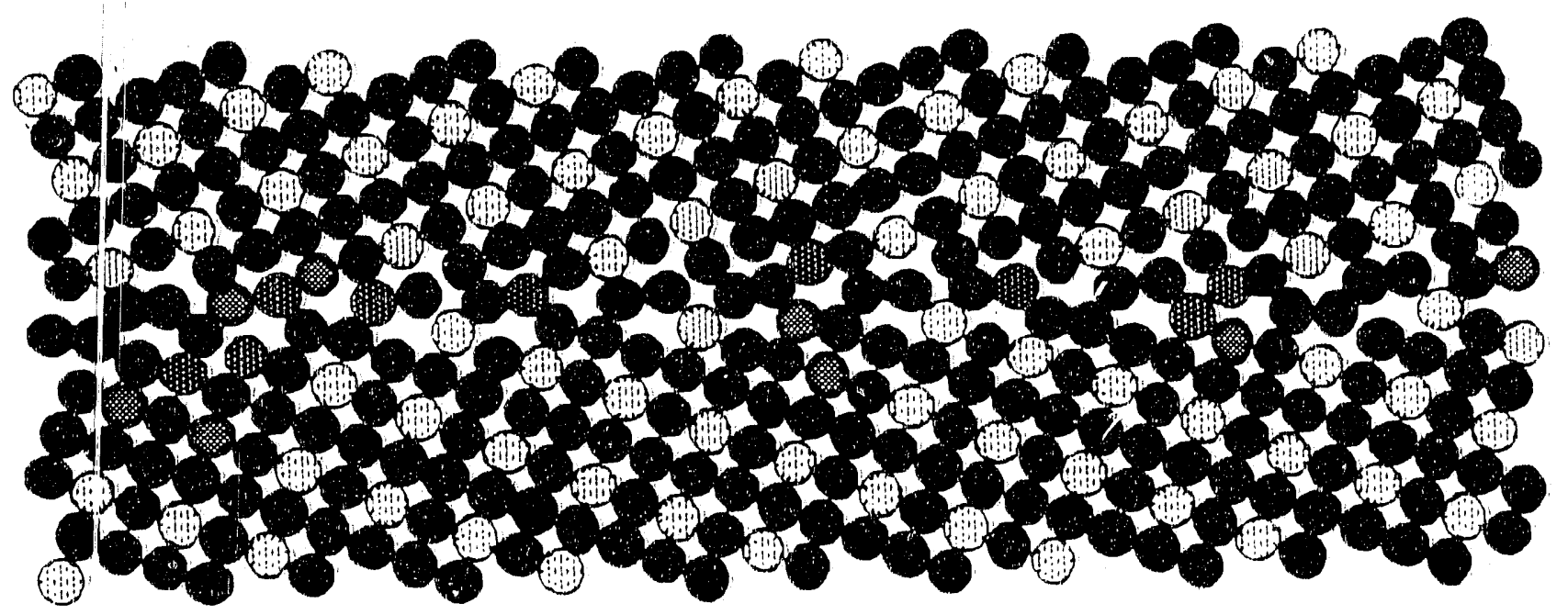

Fig. 4. Structure of the 5294520$)$ tilt boundary in the Ni rich No.24\% Al alloy.

In contrast in Al-rich alloy, Ni.26\% Al. Al segregates only to certiain well defined atomic sites and there are no interchanges hetween Ni and Al atoms. This is seen from both Fis. 3 b and 5 which show, respectively, the concentration protile of Al surplus in the grain houndary and the atomic structure in which shading rapresents the chemical nature of the atoms similarly as in Fis. $t$. No significant compositional disorder develops in the vicinity of the houndary. () m the contrary, since the segregation of aluminum is very selective, a narrow layter of a two dimensional ordered Ni.Al compound with a high aluminum content is formed at the grain boundary. This is clearly visible in Eig. 5.

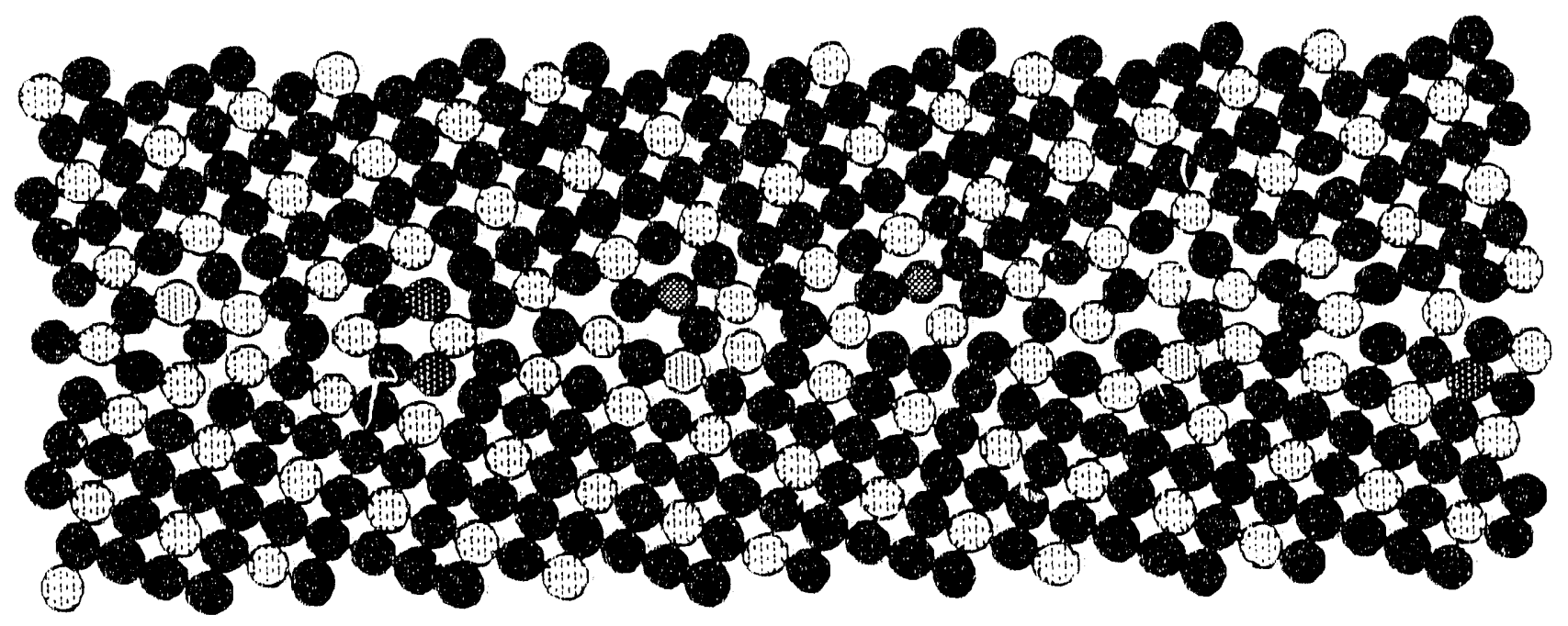

Fig. 5. Structure of the E29620) tilt boundary in the Al rich Ni.26\% Al alloy 
'The most probable reason for the segregation differences between $\mathrm{Ni}$ and $\mathrm{Al}$ is the different atomic size of these two elements. Alluminum, being a large arom, can only segregate to sites with large free volume and, therefore, its segregation is very selective. Once these sites have been filled the boundary becomes saturated. At this point a twordimensional ordered gxain bowndary structure has been formed. On the other hand, nickel is: smailler and can, therefore, segregate to a number of sites in the grain boundary. Additionally, more favorable sites are formed if further disordering occurs in the boundary region. In the case of doping by boron cosegregation may enhance the segregation of $\mathrm{Ni}$ in Ni-rich alloys which then leads to the compositional disordering of the boundary region and, consequently, to a decrease of propensity to fracture. On the other hand, segregation of aluminum in an Al-rich alloy leads to formation of a two-dimensional ordered grain boundary structure which may increase the propensity to brittle cracking. These may be the reasons why boron doping increases the grain boundary ductility only in mickel rich NigAl alloys.

\section{DEVELOPMENT OF QUANTUM MECHANICAL METHODS.}

The quantwm mechanical method which we are developing is based on the first principles tight-binding LMTO method. The main advantages of this approach are that s-, p-, d-and f-electrons are all breated on the same footing and the 'hopping' integrals are very short ranged in the non-orthogonal representation and, therefore, transferable. The Hamiltonian is written as

$$
H=\mathbf{C}_{\beta}+\Delta_{\beta}^{1 / 2} S_{\beta} \Delta_{\beta}^{1 / 2}
$$

where $C_{\beta}$ and $\Delta_{\beta}^{1 / 2}$ are diagonal matrices of potential parameters which depend only on the element considered and $\mathbf{S}_{\beta}$ is the matrix. (generally non-diagonal) of structure constants which depends only on atom positions. (If two species are present in the assembly two different values of $C_{\beta}$ and $\Delta_{\beta}^{1 / 2}$ are used when evaluating the Hamiltonian). The total energy of the system can be written as

$$
\mathbf{E}_{\text {tot }}=\mathbf{E}_{\text {band }}+\mathbf{E}_{\text {rep }}
$$

where the first term is the attractive band energy and the second term describes the repulsive interaction arising from the double counting and exchange and correlation intexactions. The latter can be approximated by a repulsive pair. 
potential which is wswally titted semi-empirically, for example so, as to reprodice the correct equilibrium density and the bulk modulus of an appropriate ideal lattice.

This: scheme would be a classical tight-binding method if all the potential parameters were fixed and the structure constants corresponded to two-center canonical hopping integrals which vary with the separation of atoms and the orientation dependence of which is given by the Slater-Koster relations. In the presemt development two important improvements have been incorporated into the method. First the structure constants are evaluated beyond the two-center approximation and are, therefore, determined by the overall configuration oc the atomic cluster considered. Secondly, the potential parameters are taken as functions of the local density which is determined independently by fully selfconsistent calculations of ideal lattices at different densities. Notwithstanding, in any such scheme very unphysical charge distributions may be attained in non-ideal structures since the charge density and the corresponding Hamiltonian are not selfconsistent. This problem has been frequently overcome by imposing the condition of orbital and atomic charge neutrality as a replacement for self-consistency. This is the condition that determines the site diagonal elements of the Hamiltorian. However, this is certainlly inappropriate in the case of alloys since charge trar sfer is. one of the main features of alloy formation and most probably even in the cores of defects in mono-atomic solids. One of the major advantages of our scherne is then that charge neutrality is not imposed but a Dyson-like equation links the site diagonal and off-diagonal elements of the matrix $S_{\beta}$ and replaces the charge neutrality condition.

However, owing to the atomic sphere approximation (ASA) used in the development of the method, changes in the band energy associated with distortions which do not lead only to an overall change in the density (for example a shear deformation), are not well represented. At the same time a proper account of such distortions is essential for application to crystal defects, in particular interfaces. Two alternative treatments of this problem are being developed and tested at present. The first involves addition of another semiempirical term, $E_{A S A}$, to the total energy which contributes only in the case of shear distortions but not density changes. The functional form of this term was derived in the framework of the effective medium approximation. In the second alternative the electrons in the interstitial space between the spheres are included explicitly but approximately into the calculation of the total energy. In our development this is done by means of the so-called warping correction. Both these schemes require empirical input which 
can employ available experimental data (for example elastic constants) or results of ab-initio electronic structure calculations of the total energy. For this purpose the full potential self-consistent calculations are used, analogously as when constructing the copper-bismuth potentials.

\section{RECENT PUBLICATIONS}

V. Vitek and S. F., Ch. M: Modeling of grain boundary structures and properties in intermetallic compouads, Scripta Mietall: 25, 1237, 1991.

V. Vitek, G.J. Ackland and J. Cserti: Atomistic Modeling of Extended Defects in Alloys, in Alloy Phase Stability and Design, MRS Symposium Proceedings, Vol. 186, p.237, 1991.

V. Vitek: Micromechanisms of Intergreaular Brittle Fracture in Intermetallic Compounds, J. Phys. Paris III 1, 1085, 1991.

D. E. Luzzi, Min Yan, M. Sob and V. Vitek: Atomic Structure of a Grain Boundary in a Metallic Alloy: Combined Electron Microscopic and Theoretical Study, Phys. Rev. Lett. 67, 1894, 1991.

Min Yan, V. Vitek and G. J. Ackland: Atomic Structure and Chemical Composition of Grain Boundaries in $\mathrm{L1}_{2}$ Intermetallic Compounds: Relation to Intergranular Brittleness, Proc. NATO Workshop on Intermetallic Compounds, Irsee, in press, 1992.

Min Yan, V. Vitek and G. J. Ackland: Structure of grain boundaries in $\mathrm{L1}_{2}$ alloys at finite temperatures: Effect of deviations from stoichiometry, in ?? MRS Symposium Proceedings, in press, 1992.

Min Yan, M. Sob, C. J. Ackland, D.E. Luzzi, V. Vitek, M. Methfessel, C. O. Rodriguez and $\mathrm{O} . \mathrm{K}$. Andersen: Interatomic forces and atomic structure of grain boundaries in copper-bismuth alloys, Phys. Rev., to be published, 1992.

V.Vitek, G.J.Ackland, M. Menyhard and Min Yan: Atomic Structure of Grain Boundaries in Ordered and Disordered Binary Alloys, Indo - U.S. Symposium on Structure and Properties of Interfaces, Bangalore, India, in press, 1992. 

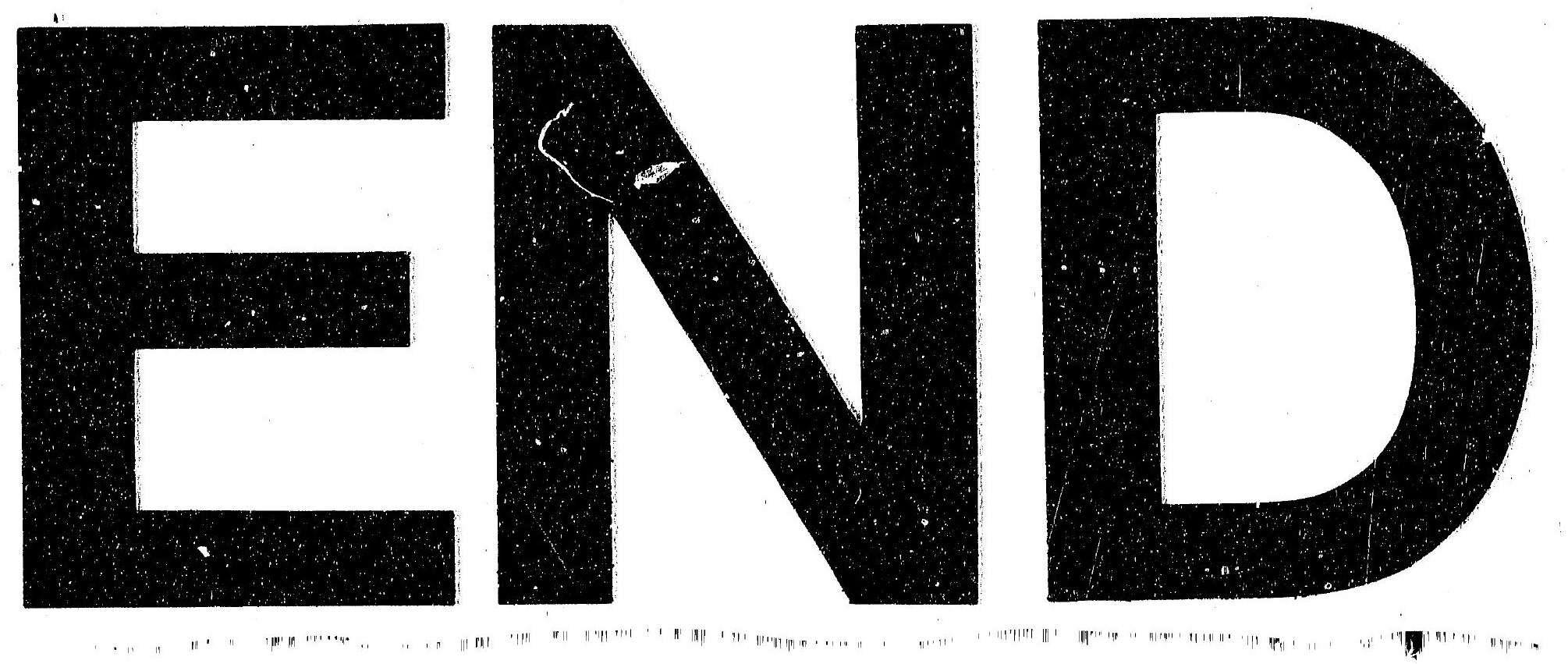


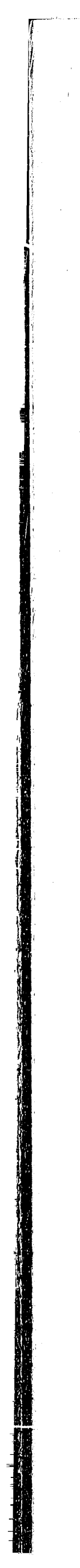

